\author{
Maria Raquel Pozzio
}

Universidad Nacional de La Plata, Ensenada, Provincia de Buenos Aires, Argentina

\title{
La gineco-obstetricia en México: entre el "parto humanizado" y la violencia obstetrica
}

\begin{abstract}
Resumen: Al mismo tiempo que la gineco-obstetricia se feminiza, aumenta la problematización de la violencia obstétrica. Es a partir de estos dos fenómenos que el artículo se propone describir las diferentes posturas que los actores han tomado en torno de la introducción de prácticas de 'parto humanizado' - clasificadas como 'abiertas', 'intermedias' y de 'resistencia'-, en tanto y en cuanto éstas buscan disminuir la violencia obstétrica. En especial, se subrayan las explicaciones y reacciones de las mujeres gineco-obstetras ante la violencia obstétrica. El análisis busca mostrar cómo el género como construcción y la antropología como método y enfoque contribuyen a entender el fenómeno y hacer inteligible esta aparente paradoja. Como conclusión, se plantea que establecer relaciones entre la feminización de la ob-gyn y la problematización de la violencia obstétrica nos permiten una entrada a la comprensión de los alcances y limitaciones del proceso de feminización de la profesión médica.
\end{abstract}

Palabras clave: género; medicina; parto; violencia obstétrica; antropología

\section{(c) (†)}

Esta obra está sob licença Creative Commons.

'Usaré las comillas simples para dar cuenta de los términos tal como han sido utilizados por los actores en el trabajo de campo, algo que en antropología llamamos términos 'nativos'. Los nombres reales de los informantes han sido modificados. ${ }^{2}$ Las mujeres que realizaron la especialidad de Gineco-Obstetricia y obtuvieron su certificación ante el Consejo Mexicano de Ginecología y Obstetricia representaban hasta 2011 el $39 \%$ del total de los médicos certificados, el porcentaje más alto de mujeres

\section{Introducción'}

En las últimas décadas se ha dado un proceso de feminización de la profesión médica en México que tiene rasgos peculiares según la especialidad. Así, segmentada horizontal y verticalmente, la feminización es notable en algunas especialidades, como la gineco-obstetricia. ${ }^{2}$ De manera concomitante, esta especialidad, señalada por tantos autores como uno de los principales dispositivos científicos producidos con la finalidad de controlar el cuerpo y la sexualidad de las mujeres, ${ }^{3}$ ha comenzado a problematizar lo que diferentes actores sociales han nominado como violencia obstétrica, problematización que no puede desvincularse de la promoción del parto humanizado. ${ }^{4}$ Las propuestas y debates en torno del parto humanizado - o natural, fisiológico y sus diversas acepciones 
certificadas por ese consejo en la historia del mismo. En otro artículo, sostenía que "es de esperar que estos porcentajes vayan en aumento dado que, entre quienes aprobaron su examen de residencia para entrar a Ginecología y Obstetricia en 2011 , el $63 \%$ eran mujeres" (Pozzio, 2014). Las cifras de 2011 fueron las que me brindó el CIFRHS ante un pedido especial, ya que los resultados de los exámenes de residencia que se publican año a año no permiten saber el sexo del/ de la residente. Consultar los registros de los médicos y médicas asociados a Colegio Mexicano de Especialistas en Ginecología y Obstetricia permite una idea aproximada de la proporción de varones y mujeres en la especialidad. Véase: http:// www.comego.org.mx/index.php/ comegomenu/colegiados/ titulares.

${ }^{3}$ Fabiola ROHDEN, 2002; Marcela NARI, 2004; Oliva LÓPEZ SÁNCHEZ, 2010; Agustina CEPEDA y Cecilia RUSTOYBURU, 2014.

${ }^{4}$ Con "parto humanizado" estoy utilizando los mismos términos de los actores, que funciona de sinónimo de parto fisiológico desmedicalizado, natural, respetado, entre otros. Hace referencia a las distintas modalidades que buscan desmedicalizar el parto y hacer respetar los derechos de las mujeres y las familias en el momento del nacimiento de un bebé.

${ }^{5}$ Graciela FREYERMUTH y Paola SESIA (coords.), 2009.

${ }^{6}$ Desde marzo de 2012, fecha de su aplicación, no se han registrado hechos relacionados "lo que pone de manifiesto que la existencia de un tipo penal de violencia obstetrica plantea desafios en su persecucion y que -al menos en Veracruz- no esta siendo aplicado". Informe GIRE, 2013 , p. 42.

${ }^{7}$ Franco ROSALES, 2013.

${ }^{8}$ Roberto CASTRO y Joaquina ERVITI, 2014.

${ }^{9}$ Beverly CHALMERS, 1992.

10 "Ahora es casi universalmente reconocido que a menos de que los practicantes tradicionales de
- en México, están atravesadas por las reivindicaciones y demandas de los movimientos y asociaciones de mujeres, feministas, indígenas y de derechos humanos; pero también, y sobre todo desde el ámbito académico y la política pública, se lo ha venido promoviendo como estrategia que ayude en la disminución de la mortalidad materna. En esta última línea se ha insistido en recuperar los saberes de las parteras empíricas indígenas como agentes capacitados y articuladores entre la comunidad y los servicios de salud, sobre todo por su papel fundamental en los estados con gran población indígena, que son al mismo tiempo los que tienen los índices más elevados de muerte materna, como Chiapas, Guerrero y Oaxaca. ${ }^{5}$ Así, si bien la Ley de Acceso de las mujeres a una vida libre de violencia no incluye en sus definiciones a la Violencia Obstétrica, las leyes locales de Chiapas, Guanajuato, Durango y Veracruz sí la han contemplado - en esta última entidad, la Violencia Obstétrica está tipificada como delito; ${ }^{6}$ al mismo tiempo, la Norma Oficial Mexicana 007, de Atención de la Mujer durante el embarazo, parto y puerperio y del recién nacido, fue modificada en 2005 "para buscar disminuir los daños obstétricos y los riesgos para la salud de las mujeres y de sus hijos". ${ }^{7}$ Casos resonantes que saltan a la prensa han puesto de relieve en el último tiempo la Violencia Obstétrica, más que nada - como señalan Roberto Castro y Joaquina Erviti como violaciones a los derechos reproductivos y sexuales de las mujeres. Todo esto, en un contexto donde la cuestión de los derechos humanos en México está siendo disputada y discutida públicamente; y que muestra que las políticas llevadas a cabo para resolver o disminuir el problema, reduciendo la Violencia Obstétrica a un problema de "calidad de la atención", han fracasado. ${ }^{8}$

La situación de México no es ajena al contexto mundial: en los últimos treinta años la lucha entre el nacimiento como problema médico y el nacimiento humanizado se ha intensificado y globalizado. En este marco, la OMS ha lanzado diversos documentos y declaraciones, entre las que se destacan las recomendaciones de $1985,{ }^{9}$ que vuelven a poner en el centro de la escena a las mujeres, tanto a las parturientas, como a las encargadas de ayudar a parir, cuya exclusión de la práctica legal ha sido considerada como un gran error. ${ }^{10}$

En América Latina, luego del Congreso "Humanización del Parto y el Nacimiento", realizado en Ceará, Brasil, en noviembre del año 2000 , se formó la Red Latinoamericana y del Caribe para la Humanización del Parto y el Nacimiento (RELACAHUPAN), que agrupa y pone en contacto a redes nacionales, agrupaciones y personas, y "que propone mejorar la vivencia del parto y la forma de nacer". Esta red 
la salud (incluyendo a las parteras) sean apropiadamente reconocidos y articulados dentro de sistema nacional de salud, los países nunca podrán logra cobertura adecuada de salud para toda su población. Casi en todas partes, los intentos por aumentar las destrezas y conocimientos de las parteras tradicionales y de movilizarlas para que jueguen un rol efectivo dentro del sistema de salud se han encontrado con la oposición de los grupos organizados de profesionales en salud con gran interés en mantener el statu quo. El desarrollo de una política favorable hacia la partería tradicional depende de la comprensión progresista de la naturaleza de esta atención, as como del rol y recursos de sus practicantes, muchas de las cuales poseen un caudal de sabiduría, conocimiento $y$ experiencia que solamente puede servir para mejorar la calidad de la atención que los países brindan a sus poblaciones. Las parteras tradicionales constituyen una reserva importante $y$ valiosa de recursos humanos que deben ser optimizados dentro de servicio de salud de cada país, si es que el estatus de salud de la población va a ser mejorado. Los gobiernos deben reconocer $y$ legitimar formalmente la práctica de las parteras tradicionales" citado en Marsden WAGNER 2007.

11 RELACAHUPAN- Uruguay. "Parto Humanizado: Recopilación de folletos y artículos sobre el tema. Materiales de apoyo para talleres de capacitación. 20006-2007."

12 Celia IRIART, Howard WAITZKIN Jaime BREHIL, Alfredo ESTRADA Emerson MERHY, 2002; Giglio PRADO y Verónica GONZÁLEZ, 2014

13 Mario BRONFMAN y Roberto CASTRO, 1989; CASTRO Y Alejandra LÓPEZ, 2010.

${ }^{14}$ Durante mi trabajo de campo en México me llamó la atención que las profesionales se llamaran a sí mismas "médicos" y sólo usaran el femenino cuando se es la que ha motorizado que en mayo se organice, en todos los países de la región, la "Semana mundial por un parto digno y respetado"." Si bien hay profesionales de la salud y planificadores de política pública que participan de estas redes, es interesante mencionar que salvo excepciones, como algunas voces dentro de la medicina social/salud colectiva latinoamericana, ${ }^{12}$ en general, los y las profesionales médicos han tenido una postura ajena - y a veces, hasta crítica - de las prácticas del parto humanizado, así como de toda práctica que pareciera atentar contra su posición de poder hegemónica en el campo de la salud..$^{13}$ Es en este marco que en el siguiente artículo me propongo analizar las posturas que distintos "médicos" 14 que trabajan en la atención de la salud de las mujeres han tenido en torno de las prácticas que, en el marco del Modelo de Atención intercultural del Parto, buscan "humanizarlo"15 como forma de disminuir la Violencia Obstétrica.

Señalar la contemporaneidad entre estos dos fenómenos - feminización y violencia obstétrica - no implica establecer a priori una relación de determinación, pero sí intentar resaltar contextos que pueden volverse significativos y hasta paradójicos, tanto desde el sentido común - que también contribuye a la formación del sentido común académico - como desde los sentidos o categorías de los propios actores sociales (o nativos). ¿Por qué significativos? Porque los actores del campo no se han cansado de señalar, a veces con indignación, otras con resignación, que son las propias mujeres gineco-obstetras quienes más frecuentemente ejercen la Violencia Obstétrica. Esa indignación, esa resignación, la producción de una opinión generalizada en un contexto determinado aporta sentidos -no unívocos, claro está-que resulta pertinente indagar. ¿Por qué paradójicos? ${ }^{16}$ Porque si una paradoja es "una idea extraña u opuesta a la opinión común", es interesante pensar cómo el hecho de que las mujeres sean señaladas como más violentas que sus pares varones desafía ideas comunes del universo de género dominante, que ha construido a lo femenino como lo no violento, las mujeres como sinónimo de paz, vida, etcétera. ${ }^{17}$

Por todo esto es que en este artículo trabajaré desde una perspectiva que aúna el abordaje antropológico y el análisis de género. El abordaje antropológico, respecto a analizar en contexto, los puntos de vista de los actores; el análisis de género, respecto a poner en relación el caso con las disputas de poder y significación en torno de lo femenino y lo masculino - aquí, incrustadas en el seno de prácticas biomédicas.

Los materiales con los que me propongo llevar a cabo la indagación fueron obtenidos durante una investigación donde se estudiaron las configuraciones de género en la 
llamaban "doctoras". Es por eso que aquí uso el masculino, y el entrecomillado para dar cuenta de que trata de un uso de los actores.

15 Si bien la cuestión de la humanización de la atención biomédica es un tema amplio y complejo, tanto desde su conceptualización como respecto a la planificación de la política pública, es importante señalar que si bien quienes propusieron el "Modelo de atención intercultural del parto" consideraban al mismo como parte de una crítica general a la biomedicina como deshumanizante, la posibilidad de concretarlo en un programa de salud se dio -no como en otros países en el marco de una reforma o discusión general del sistema sanitario (como por ejemplo en Brasil, ya como lo plantean Benavides y Passos) sino en el marco del reconocimiento de la diversidad intercultural y las prácticas de las distintas medicinas indígenas del país, reconociendo con ello un repertorio de acción política y discursiva posible y la existencia efectiva de espacios estatales para realizarlo -el modelo lo implementó una agencia estata cuya área de incidencia se relaciona con la medicina tradicional e indígena.

${ }^{16}$ La definición de paradoja del diccionario de la Real Academia Española que se trata de "una idea extraña u opuesta a la común opinión y al sentir de las personas" Real Academia Española http://lema.rae.es/drae/ ?val=paradoja

${ }^{17}$ Françoise HÉRITIER, 2008 ; Lucía RAYAS VELASCO, 2009

18 ROHDEN, 2002, p. 105.

19 He entrevistado a 5 ginecoobstetras mujeres con residencia, 5 doctoras que sin haber hecho esa residencia, se dedican en la práctica a la atención de la salud de la mujer y 3 entrevistas a médicos gineco-obstetras varones. Todo ello a lo largo del año 2012 en la Ciudad de México. medicina mexicana en el contexto de su feminización. En dicho estudio se constató la dispar feminización de las distintas especialidades médicas y se profundizó en lo que sucedía al interior de una especialidad en concreto: la gineco-obstetricia. La misma fue elegida considerando lo significativo de sus prácticas y saber técnico respecto a la salud y el cuerpo de las mujeres, tanto que Rohden la llama "una ciencia de los atributos esenciales de la mujer y de su naturaleza específica". ${ }^{18}$

Con el primer acercamiento a los y las profesionales que se desempeñan en la gineco-obstetricia, ${ }^{19}$ supe de un modelo de atención de partos que se estaba desarrollando desde la Secretaría de Salud del Gobierno Federal, ${ }^{20}$ uno de cuyos objetivos era estimular prácticas que apuntaran a disminuir la Violencia Obstétrica. ${ }^{21}$ Luego, conocí a un grupo de médicos del Instituto Nacional de Perinatología que también estaban preocupados por hacer de sus propias prácticas, durante el parto sobre todo, algo "más humano". Por último, en un congreso de la especialidad, se desarrollaban unas mesas redondas que giraban en torno de los "Desafíos y debates actuales en la Ginecoobstetricia". Una de esas conferencias se llamaba "¿Parto o cesárea?" y en ella se platicó especialmente de las nuevas prácticas del "parto humanizado". Estos hechos que menciono se convirtieron en "datos" importantes para pensar las tensiones y dilemas que vive una especialidad médica en proceso de feminización.

Si partimos de considerar que la medicina como saber, práctica y profesión, fue un instrumento de maternalización de las mujeres y medicalización de la reproducción y difusor del modelo binario de los sexos de la "mujer hormonal", ${ }^{22}$ considero válido preguntar si la feminización puede reducirse a un proceso demográfico o si, en un contexto de demandas de los colectivos feministas y de mujeres, la feminización puede entenderse también como proceso social que permea a la profesión médica - y, en este caso, a la gineco- obstetricia - y puede traer consigo ciertas modificaciones valorativas, simbólicas y/o materiales. Una forma de buscar operacionalizar una posible respuesta - provisoria, conceptualmente situada - puede ser indagar en cómo fue vivida e interpretada por los hombres y mujeres que se dedican a atender la salud femenina la implementación de un modelo de atención que luchara contra la Violencia Obstétrica.

\section{Partos y Violencia}

Si bien la Organización Mundial de la Salud (OMS) ha planteado que las cesáreas no deberían sobrepasar el 
${ }^{20}$ Durante el sexenio 2006-2012. ${ }^{21}$ Desde el punto de vista de mi análisis, la Violencia Obstétrica es parte constitutiva de la ginecoobstetricia como dispositivo de saber-poder biomédico. Tal como es problematizada por los actores sociales del campo, los usos y abusos de tal dispositivo son definidos como V.O., lo que desde mi punto de vista sería violencia institucional -ya que son estos contextos institucionales, atravesados por lógicas de género, clase y etnia, los que hacen posible esos "usos y abusos".

22 Thomas LACQUEUR, 1994; NARI, 2004; CEPEDA y RUSTOYBURU, 2014 .

${ }^{23}$ Los datos provienen de la Encuesta Nacional de Salud y Nutrición ENSANUT/Instituto Nacional de Salud Pública, 2012, la última realizada cuyos datos haya sido procesados y publicados y que están disponibles para el Global Health Observatory Data Repository de la OMS (http://apps.who.int/gho/ data/node.main.REPWOMEN39?la $\mathrm{ng}=\mathrm{en}$ ) Para constatar el aumento se estima que en el periodo 19882000 los nacimientos en instituciones privadas sobrepasaron la mitad de los nacimientos (sobre un total analizado de 992 casos); mientras que en 1997 las cesáreas fueron el $48 \%$ de los nacimientos, en 2003 fueron casi el 64\%. En las instituciones públicas también la operación cesárea (OC) aumentó de manera importante. Tanto en la instancia encargada de asegurar a los trabajadores del sector privado (Instituto Mexicano del Seguro Social, IMSS), como en aquella que brinda cobertura a los trabajadores del sector público (Instituto de Seguridad y Servicios Sociales de los Trabajadores del Estado, ISSSTE), la tasa de OC pasó, respectivamente y tan sólo en una década, de 21.8 a $35.2 \%$ y de 30.1 a $48.1 \%$. Por otro lado, los servicios que la Secretaría de Salud ofrece a la población abierta mantienen niveles menores de crecimiento (15.8 a $21.4 \%$ ), aunque son significativos. (Lourdes CAMPERO et al, 2007).
$15 \%$ del total de nacimientos, la realidad es que en México, como en muchos otros países de América Latina, la tasa de cesáreas sobrepasa esa cifra y sigue un constante aumento. En la República Mexicana, los datos para 2012 indicaban que poco más del $45 \%$ de los nacimientos fue por cesárea. ${ }^{23}$ Este aumento no se ha dado sin debate, tanto a nivel de los profesionales de la salud como de la sociedad civil. Como ya fue dicho, mientras distintas organizaciones denuncian los excesos de la medicalización del nacimiento y debaten y difunden las prácticas alternativas de 'parto humanizado' - que son diversas y no siempre van contra lo biomédico, ${ }^{24}$ desde el campo de la salud y los derechos humanos ha comenzado a plantearse que el aumento en el número de las cesáreas no es ajeno a un fenómeno más general que englobaría el uso y abuso de las cesáreas y toda una serie más de prácticas biomédicas institucionalizadas y violentas: uso y abuso de fórceps, oxitocina, rasurados, edemas etc. Si se considera que estas prácticas y técnicas se dan en el marco de relaciones asimétricas de poder, que generan maltrato, se comprende el sentido abarcador de lo que se denuncia como Violencia Obstétrica.

En este contexto se ha comenzado a problematizar la cuestión tanto desde lo jurídico, como el ya citado ejemplo de la Norma 007 y la ley de Veracruz, como desde lo profesional y lo estatal. Hasta 2012, la información sobre quejas médicas de la Comisión Nacional de Arbitraje Médico (CONAMED) no daba cuenta del sexo del médico denunciado, aunque se destacara que era notorio el número de especialistas de gineco-obstetricia denunciados. También, la Comisión Nacional de Derechos Humanos ha comenzado a recabar quejas y denuncias de este tipo..$^{25}$

Es en este marco donde deben comprenderse los intentos por definir y especificar la violencia obstétrica, para, a partir de ello, elaborar planes y programas que busquen reducirla o eliminarla, un grupo de médicos varones, formados en la escuela de la medicina social latinoamericana, y que estuvieron en contacto con colegas de Sudamérica que alentaron en sus propios países el parto humanizado, fueron los que diseñaron e implementaron el modelo de atención intercultural del parto de la Secretaría de Salud. Definieron la Violencia Obstétrica como un tipo de violencia que afecta la salud reproductiva y que está "frecuentemente presente en la atención convencional de los servicios institucionales y privados". ${ }^{26}$ Esta frecuencia en la atención "convencional", los alerta sobre la "naturalización" que se hace de estas violencias institucionales. La Violencia Obstétrica es entonces para ellos "producto del patriarcado, que se manifiesta en la legitimación de procedimientos como la apropiación del cuerpo de la mujer y un trato 
${ }^{24}$ Fernando MINO, 2012

${ }^{25}$ CASTRO y ERVITI, 2014.

26 José Alejandro ALMAGUER GONZÁLEZ, Hernán GARCÍA RAMÍREZ y Vicente VARGAS VITE, 2010, p. 2.

${ }^{27}$ ALMAGUER GONZÁLEZ, GARCÍA RAMÍREZ Y VARGAS VITE, 2010 p.5.

${ }^{28}$ Germán FAJARDO-DOLCl et al. 2009 . deshumanizador y una patolo-gización de sus procesos naturales". ${ }^{27}$ Es interesante insistir con que quienes escribieron esta definición son ellos mismos médicos gineco-obstetras, que por distintas circunstancias tienen una visión crítica de su formación, sobre todo la obtenida en las residencias médicas. También resaltar que son ellos mismos los que publican y los que implementan el programa, por lo que su aporte más que académico debe considerarse una intervención y una toma de posición política en los campos de disputa de la especialidad y la profesión.

\section{La violencia obstétrica y el parto humanizado: el punto de vista de los propios especialistas}

Los datos señalan a los especialistas de la ginecoobstetricia a la cabeza de las quejas de los pacientes y usuarios del sistema de salud. ${ }^{28} \mathrm{~A}$ su vez, mis informantes, tanto usuarios/ias como médicos y médicas, señalaron una y otra vez anécdotas y dichos frecuentes que ilustraban el modo en que los gineco-obstetras, y sobre todo las ginecoobstetras, ejercían lo que ha pasado a denominarse como Violencia Obstétrica. En algunos casos, se mencionan prácticas concretas, como pellizcos en la vulva o palabras obscenas dichas por el personal de salud a la parturienta. Otras veces, toda práctica gineco-obstétrica desarrollada en el seno de un hospital es vista como formando parte de la violencia obstétrica, por ejemplo, el parir en forma horizontal en una camilla. Esta tensión o polisemia existe y, siguiendo el punto de vista de los actores, consideramos que enriquece el análisis, ya que la misma ayudará a comprender las distintas visiones que se dan a favor o en contra del 'parto humanizado'.

Para sistematizar estas distintas visiones, mostraré las posturas de un grupo de médicos y médicas gineco-obstetras ante el parto humanizado, posturas que se dieron en el marco de la implementación de un programa denominado "Modelo intercultural de atención del parto", de la Secretaría de Salud del Gobierno Federal, en el sexenio 20062012. Propongo que las diferentes posturas de los y las profesionales ante el programa y las prácticas que introduce, son una vía de entrada útil a la comprensión de las contradicciones y paradojas que viven estos doctores y doctoras, particularmente éstas últimas, en el contexto de feminización de la especialidad.

Así, enfrentados a las resistencias de la 'corporación gineco-obstetra', ${ }^{29}$ pero a la vez conscientes de que para hacer realidad el programa era necesario trabajar con sus propios colegas, buscaron profesionales que pudieran 
'llegar' a otros/ras gineco-obstetras sin serlo necesariamente, y formarlos y capacitarlos en las prácticas de 'parto humanizado'. Para ello, contrataron a médicos generales y homeópatas, como el caso de la doctora Yola, médica oaxaqueña, de unos cincuenta años:

Yo decía no se trata de pelear con ellos, de enjuiciarlos tanto, son esto, son lo otro, porque pues están defendiendo tantos años que les costó o nos costó: una carrera de medicina es... no es fácil, y la especialidad no es fácil, entonces, les cuesta soltar todo eso [...] Pero si uno logra contactarlos y exponerles el por qué y cómo, es más bien invitarlos a que escuchen, más que atacando o confrontando. Una de ellas, una de las "ginecos" me decía "por qué nos atacan tanto" "por qué no nos escuchan también" Entonces, yo lo que fui aprendiendo de ellos era pues, a invitarlos...

La doctora Yola insiste que para modificar la práctica no hay que ir 'contra' los gineco-obstetras, sino que hay que seducirlos, convencerlos, y también comprenderlos. Su experiencia como promotora del 'parto humanizado', le permitió clasificar las posturas de los 'ginecos' del siguiente modo: los más abiertos, que se dejan enseñar y ponen en tela de juicio sus propios saberes y prácticas; los que buscan una salida negociada entre su poder, autoridad y saber y los derechos de las pacientes; y los que de plano, se resisten. Adoptaré esta clasificación porque es una clasificación "nativa" que resulta, por lo demás, muy ordenadora.

\section{Los más abiertos}

Sobre los 'más abiertos', la doctora Yola narraba:

Hubo unos "ginecos" que me tocó, y que estuvieron muy atentos. En uno de los hospitales tuvimos la práctica y una de ellas, una "gineco" decía -"Doctora, yo me siento aquí, yo voy a estar calladita, sentadita $y$ me va a enseñar cómo" $Y$ entonces estuvimos mostrándole los pasos y lo que decía ella era -"ipor qué nunca nos enseñan esto?" - "qué bonita atención, por qué nunca nos enseñaron esto, sino que tenemos prácticas erróneas" ¿no? Entonces, estaba admirada, del tipo de atención que le mostrábamos.

Pude identificar esta misma posición en algunos integrantes de un grupo de residentes del Instituto Nacional de Perinatología, quienes estaban muy interesados por conocer protocolos e información que les permitiera introducir algunas cuestiones relacionadas con el 'parto humanizado' en una institución a primera vista tan difícil para ello como 'Perinato' - hospital de tercer nivel, donde se 
atienden un gran número de embarazos de riesgo. Uno de los residentes, un joven graduado de la UNAM que estaba especializándose en medicina reproductiva, planteaba que estar o no abiertos a estas cuestiones no dependía tanto del género y sí más de la edad, por lo que él tenía esperanzas de que las generaciones más jóvenes fueran más permeables al 'parto humanizado'.

\section{Las resistencias}

Para ilustrar las posturas más duras, de aquellos que se resistían, la doctora Yola narró, en la entrevista que le realicé en su consultorio, el modo en que muchos colegas se paraban y retiraban de las pláticas que ella brindaba, y lo hacían con alevosía, queriendo mostrar que nada de lo que se decía les interesaba. Sin embargo, creo que ni esas posturas muestran las resistencias más radicales, que yo misma como etnógrafa pude conocer durante mi trabajo de campo, cuando asistí como oyente a una mesa redonda llamada "Tendencias en Ginecología y Obstetricia iregresión o progreso?" en el Congreso Regional del Colegio Mexicano de Ginecología y Obstetricia, realizado en la ciudad de México en junio de 2012. Baste como ejemplo, la conferencia de un renombrado ginecólogo, sexagenario, titular de cátedras de la UNAM y con un papel muy activo en las corporaciones médicas del país y la Región - a quien llamaremos el doctor Quintana - en una postura de defensa de las cesáreas que trataba las Recomendaciones de la OMS para el parto y el nacimiento como un material inservible y se dirigía, beligerante, hacia su auditorio del siguiente modo:

¿qué significa un parto humanizado? Vean esta lista interminable de la OMS (Lee) "favorecer la libertad de posición y movimiento -trabajo de parto; en cuclillas, en el agua, semi sentada o como lo deseen" ¿Ustedes saben atender partos en cuclillas? ¿Les ha tocado esto? No me digan que no, cuando llega una señora y cae en rodillas y hay que atender el parto para... -pausa...entonces sí saben (Risas del público) (Sigue leyendo siempre en tono burlón) "Promover el vínculo personalizado, rescatar la necesidad de atención de la mujer de ser acompañada de su familia" ihasta sus amigos! (exagerando) Imagínense ahí... itodo un show! (cínico) Perfectamente (Sigue leyendo) "cuidar del vínculo inmediato del recién nacido" o sea, no cortar el cordón; que no hay que hacer exámenes innecesarios, todos los exámenes eso que hacen de que le pican al pobre chamaco los pediatras, algo tienen ellos contra esto. Siguiendo: La OMS, aquí, dijo "admisión espontánea, deambulación, tener un apoyo emocional" o sea una doula, escoger el tipo de mesa 
${ }^{30}$ Por ejemplo, "el parto vaginal está bien para una sueca que tiene un cabus (cadera o cola, en caló mexicano) de este tamaño, pero no le pidamos igual a una oaxaqueña desnutrida por generaciones" dixit del Docto Quintana. de parto iYa me imagino! "¿Qué versión quiere usted señora? En este lugar tenemos tres, escoja usted (Risas del público a partir de aquí in crecendo, también porque el tono de exposición del doctor Quintana se va volviendo más grotesco)" Éstas son las recomendaciones, vean ustedes: "valorar canalizar una vena, evitar oxitocina, permitir el parto espontáneo" nada de "puja y ahorita mijita" cuando ella quiere y como quiera, que la episiotomía no sea rutinaria... al fin, y las que hay que evitan los desgarros, pero... "Alumbramiento expectante" por eso, ayer decía yo, que un porcentaje de las pacientes que se atienden en su casa, ante el alumbramiento expectante iacaban el $36 \%$ en el hospital! ahí esperando, hasta que se cierra el cuello y a ver cómo lo sacas, y a ver cómo le sacas a pedazos y a ver cómo lo exploras, y a ver cómo le sacas lo que tiene y luego le dejas un cacho de placenta. Las suturas de desagarro no van a ser fáciles. Y favorecer a la lactancia, pues nosotros no estamos peleados con la lactancia ¿Alguien está peleado con la lactancia? Tal vez algunos hasta la promueven o son amigos de la leche... Otros no han dejado de lactar (Risas).

Y respondiendo a las denuncias - sobre todo periodísticas, de un aumento desmesurado de las cesáreas en México - el doctor Quintana justificaba desde su tribuna:

Que hoy una serie de gentes deciden tener esta forma de nacimiento simplemente por el hecho de que es muy nice tener una cesárea y no un parto de eso, de que el niño nazca entre orina $y$ excrementos, mejor no..." [...] "Esto es una situación ¿Qué les parece? Les dice el médico —"su fecha de parto es el 17" —"estaré ocupada, prefiero el 4 a las 15:00 ¿Le parece?" Esta es otra forma de ver a las pacientes en la obstetricia ¿sí? "Tu bebé es muy valioso" ¿Cuántas veces decimos eso? "Creo que no debemos arriesgar, mejor un cortecito $y$ ya está tu bebé contigo" ¿Alguno no dice esto? Levante la mano el que no lo haya dicho ¿Nadie? Gracias.

Este doctor, que además no se inhibió de tener declaraciones racistas, ${ }^{30}$ ante un público que no se inmutó con sus dichos, deja plasmado con sus palabras, pero sobre todo con su actitud - que podría describir como belicosa la resistencia de buena parte de la 'corporación ginecoobstétrica' ante la introducción de nuevas prácticas, como las del 'parto humanizado'. Desde mi punto de vista, la fluidez de su discurso y la normal recepción del mismo pueden servir como muestra de la naturalización de la violencia obstétrica en buena parte de los y las profesionales, lo cual sustenta la idea de que está íntimamente enraizada en la especialidad y es parte constitutiva de la misma. 


\section{Posturas intermedias}

Volviendo a las clasificaciones de la doctora Yola, también es importante mostrar posturas intermedias, donde lo que se intenta es comprender y no juzgar, tal como lo planteaba la doctora Amalia, joven médica ginecoobstetra del Instituto Mexicano de Seguridad Social (IMSS), que también tiene su consultorio privado en una populosa colonia del centro del Distrito Federal:

Amalia: Es como el aborto o sea, uno tiene el derecho a elegir "quiero que nazca por abajo mi bebé" o simplemente no quiero tener dolor en su debido momento y con todas las garantías, quiero que sea vía cesárea...

Investigadora: tú platicas eso con tus pacientes... cuando ellas quieren

Amalia: por supuesto "ahora ¿cuántos bebes quieres? ¿Quieres uno, quieres dos?" o sea, ya no, ya los pacientes no pasan de dos, tres ya son bien intrépidos (les digo) que valientes ino? Pero si quieren dos las programan y puede ser cesárea y sin problema. Yo así les digo "quieres parto" pues te dejamos a parto, pero finalmente tampoco el que empecemos un parto es una garantía de que tengan un parto...

La doctora Amalia, quien tuvo a su propia hija por cesárea, realiza ambas prácticas, pero, no está demás aclarar, su idea del parto fisiológico no tiene connotaciones negativas o despreciativas -como sí podía verse en el discurso del doctor Quintana. Ella opina que:

Yo creo que el parto es completamente fisiológico, con ayuda o sin ayuda del médico el parto pues, los bebés van a nacer...todo lo demás que están tratando de hacer, es como decir, como querer vender la lluvia no podemos vender el que "te voy a garantizar" porque si la frecuencia cardíaca de ese bebé dice no, te voy hacer cesárea y ya. Entonces sí yo quiero que sea integro no lo paso por ahí, lo saco por arriba.

Otra joven doctora, pionera en la implementación en un hospital federal al sur de la ciudad de México de algunas de las prácticas auspiciadas por el modelo intercultural, reflexionaba:

Yo en alguna ocasión comentaba que eso de parto humanizado, como tal, a mí no me gusta ese concepto, porque da entender que lo que nosotros hacemos ies inhumano! iY no es así!... yo creo no es tampoco culpa tanto del ginecólogo, que todo eso se haya ido dando a lo largo del tiempo, de manera rutinaria, yo creo que es como parte de un sistema

110 Estudos Feministas, Florianópolis, 24(1): 101-117, janeiro-abril/2016 
¿eh? De todo un sistema ¿por qué? Porque por ejemplo, violencia obstétrica hay muchas formas de violencia obstétrica, hasta el hecho que tú tengas a una paciente sentada en una silla y que no le puedas ofrecer una cama digna para acostarla y para llevar su atención, en una cama o en una camilla adecuada y la tengas que tener sentada: eso es violencia. Pero eso no depende de ti, eso depende de la infraestructura del hospital, de los servicios de salud que hay...

Hasta aquí entonces, las diferentes posturas ante la introducción y fomento de prácticas de 'parto humanizado', tal como fueron clasificadas por nuestros informantes: las más abiertas -como las de quienes con gusto participan de las capacitaciones dadas por la doctora Yola; las intermedias y las de resistencia. Estas diversas posturas nos ayudan a comprender los modos con que la especialidad se enfrenta y comprende nuevos contextos y desafíos. En este marco, es importante dar un paso más y analizar las actitudes y opiniones de las algunas mujeres ginecoobstetras ante hechos abiertamente etiquetados como de violencia obstétrica. En otras palabras, ¿por qué ellas son señaladas como las 'más maltratadoras'? ¿Qué opciones tienen ante sí? ¿Cómo viven la paradoja de ser víctimas y victimarias de la violencia de género institucionalizada?

31 Anónima, 2010.

\section{Las explicaciones de ellas: "todas hemos sufrido violencia".}

Durante el trabajo de campo di con dos ejemplos distintos, que sirven para ilustrar lo que suponemos debe ser la gama de posibilidades que las mujeres médicas tienen ante sí. Uno es el caso de la doctora Caty, una joven médica que realizó el primer año de residencia en gineco-obstetricia en un prestigiado establecimiento público del área metropolitana de México y que después de ser testigo de prácticas de violencia obstétrica -e instada a participar de las mismas con naturalidad, como partes constitutivas de su formación-decidió renunciar a la residencia y hacer pública una carta donde denunciaba de forma anónima lo ocurrido, con el objetivo de sentar precedentes de un debate y una situación que debería poner en jaque, al menos, la conciencia de sus colegas. ${ }^{31}$ La doctora Caty, luego de estos hechos, partió al extranjero en la búsqueda de experiencias del "buen nacer" que su formación en gineco-obstetricia en México estaba lejos de darle.

Otro es el caso de la doctora Suza, quien, luego de terminar su residencia en gineco-obstetricia con un cuadro de burn-out, renegando de su formación pero con la convicción de que debía 'hacer algo' con esa experiencia, 
realizó una maestría en estudios de la mujer y actualmente se dedica a realizar talleres y capacitaciones en el tema. Reflexionando sobre lo que había vivido y lo que escuchaba decir a sus congéneres, la doctora Suza planteaba que:

...O sea, yo, para entrevistar a las mujeres les decía "todas hemos sufrido violencia" o sea, como para que se sintieran más en confianza, y a la fecha parte de lo que hago es dar pláticas, cursos de capacitación, entonces, les digo "todas la mujeres hemos sufrido violencia, a la que diga que no, es que todavía no se ha dado cuenta". Entonces, yo creo que mucha de la violencia que ejercen las propias mujeres contra las usuarias, tiene que ver con ... es como una violencia reactiva, es como la que ejercen con los hijos ino? O sea, me violentaron... entonces, pues me tengo que desquitar con la que está debajo de mí. O sea, si yo era el último peldaño, hay alguien más abajo...También, otra explicación que creo va de la mano es lo que mi mamá me hacía: mi mamá era una mujer violentada, y me violentaba a mí, como en este sentido de hacerte más fuerte ino? -"a ti no va pasar eso, y tú tienes que, y no puedes fallar" como "sálvate tú" aunque los métodos sean un poco crueles ino? entonces, supongo que va por ahí, quiero pensar que va por ahí.

Además de reflexionar, la doctora Suza describió sus actitudes como gineco-obstetra:

Siempre me ha parecido como un cuarto de tortura ¿no? las áreas de toco cirugía, incluso antes de saber del parto humanizado, antes de todo, las señoras ya sabes -"es que me quiero parar a caminar, es que quiero estar de pie" ino? y todo mundo -"no, no, no tiene que estar acostada" bueno, pues yo era de las que les decía "sí" y yo las ayudaba a caminar ... a la hora de pujar les decía yo -"lo estás haciendo muy bien" en vez de -"!no, así no!" ino? esta maniobra que está prohibidísima que se llama Kristeller que les aplastas la panza, y yo nunca aprendí a dar Kristeller, aprendí a poner fórceps pero nunca a dar Kristeller, entonces les ponía yo la mano en la panza y les decía -"yo te ayudo" entonces, hacía cara como de que aplastaba la panza pero obvio no ino? entonces les decía -"mira, ya está saliendo" o ese tipo de cosas. La diferencia está en que ahora, me cimbra más, o sea, yo lo vivo como más intenso, lo sigo haciendo igual y es como... desde hace mucho tiempo llegué a la conclusión de que no eres la gran doctora, de que no es que sepas mucho, es simplemente que las acompañas un ratito ino? o sea, tu papel no es saber grandes cosas, es simplemente poder acompañar a alguien y ayudarle a sentirse menos sola ino? con menos miedo.

112 Estudos Feministas, Florianópolis, 24(1): 101-117, janeiro-abril/2016 
32 LAQUEUR, 1994; ROHDEN, 2002.

${ }^{33}$ Karin JOHANNINSSON, 2006; LÓPEZ SÁNCHEZ, 2010.
Con el ejemplo que sus palabras brindan, hay una clave de entrada a la comprensión de las pequeñas acciones que quizá, aunque microscópicas, se hayan convertido en formas de vivir y transformar la aparente paradoja de ser una mujer en el seno de una especialidad que las ha cosificado, las maltrata, pero que, al mismo tiempo, se ha convertido en una de las más elegidas por las jóvenes recién graduadas; una forma de sobrevivir y de transformar, de no ejercer violencia y resistirla en silencio, desde la acción individual.

\section{Buscando responder antropológicamente a la paradoja}

Comencé este artículo planteando la concomitancia de dos fenómenos que pueden ser interpretados, a primera vista, como una aparente paradoja: la feminización de la gineco-obstetricia y la problematización, en dicha especialidad, de la violencia obstétrica. Un análisis antropológico y de género, me ha permitido dar cuenta de lo significativo de la aparente paradoja así como las posibilidades, desde el análisis de los puntos de vista de los actores, de superarla. ¿De qué forma? En primer lugar, considerando que el género se construye y que no hay características innatas o esenciales en varones y mujeres. Esto ayuda a comprender que, aunque aumente el número de mujeres en un ámbito concreto esto no lo convierte, directamente, en un ámbito más femenino. Porque además, ¿qué sería en tal caso lo femenino? Sólo una narrativa de las mujeres como víctimas - que no comparto - podría sostener que "la mujer" sólo es víctima de violencia y nunca ejecutora. Se señala que las mujeres son las que más maltratan, pero en realidad todos maltratan y hay diversidad de género entre quienes maltratan y quienes van por modelos alternativos, críticos, y/o buscan - al menos modificar la 'misoginia' enraizada en el origen mismo de la gineco-obstetricia. Quiero decir con todo esto que no existen identidades esenciales del género, pues éste se construye; es decir, lo femenino se construye y modifica. En segundo lugar:¿quiénes lo construyen? Sin dudas, un saber-poderprofesión como la medicina científica ha tenido mucho que ver con la forma en que son vistos y tratados los cuerpos, la fisiología y la sexualidad de las mujeres. ${ }^{32}$ De modo que ese saber-poder-profesión es uno de los elementos que sostiene el orden de género. Por ello, que en su seno se problematice una cuestión que antaño formaba parte del quehacer cotidiano que nadie discutía ${ }^{33}$ puede estar indicando que existen ciertos resquebrajamientos de ese dispositivo. Desde este punto de vista, la feminización puede leerse como 
${ }^{34}$ Lena SOMMESTAD, 1994; Beatriz KALINSKY, 2006; Marie BUSCATTO y Catherine MARRY, 2009

${ }^{35}$ Joan SCOTT, 2001.

36 Octavio BONET, 1999; Ana DELGADO, Anay TÁVORA y Teresa ORTIZ GÓMEZ, 2003. causa y consecuencia a la vez de ese resquebrajamiento, las grietas por las que las cambiantes relaciones de género societales permean el conocimiento y las prácticas de la medicina científica. Esto significaría considerar a la feminización no como un mero cambio demográfico, sólo de números, sino como un proceso de cambio configuracional - en lo material y en lo simbólico - del ejercicio profesional. ${ }^{34}$

Tercero y último, lo que a primera vista resulta paradojal no son más que tensiones y dilemas que encarnan en la propia experiencia de las doctoras, experiencias en tanto lo entiende Joan $\mathrm{Scott}^{35}$ - es decir, relatos que ya contienen una interpretación. Este dilema es el que ellas expresan en la vivencia contradictoria de ser mujer y ser médica, ${ }^{36}$ experiencia que al mismo tiempo enriquece, y vuelve más estimulante y densa, su práctica profesional. En esta última cuestión entra la antropología, con su vocación por lo microscópico y lo simbólico, poniendo de relieve lo que sucede en las interacciones sociales, en los gestos, los detalles. Así, si la violencia obstétrica es institucional, comprender las prácticas concretas de los médicos y médicas, las formas en que negocian, comprenden, reaccionan, sus mínimos gestos - fingir una maniobra de Kristeller, pero no aplicarla -, eso que los actores hacen en el cotidiano, en el seno de sus relaciones y estructuras, eso es lo que permite entender en profundidad el fenómeno, permitiendo alejarse de visiones normativistas que a primera vista, hubieran juzgado a los y las profesionales, condenándolos sin abordar la problemática más que a nivel superficial.

\section{Conclusiones}

En este artículo quise mostrar cómo, en el seno de una especialidad médica que se feminiza, la problematización de la violencia obstétrica obliga a pensar en las posibilidades de cambio, los alcances y limitaciones, que la masiva entrada de mujeres acarrea. Las diversas posturas de los profesionales frente a la introducción de prácticas de 'parto humanizado' y, sobre todo, las posturas de las mujeres médicas frente a la violencia, son una pequeña muestra de ello y de las supuestas paradojas en las que se ven envueltas. En este marco, siguiendo los sentidos "nativos" de los actores, mostré tres caminos de acción: el de los médicos más abiertos a reflexionar sobre sus prácticas y transformarlas; las de aquellos que resisten e insisten en una gineco-obstetricia que deviene violenta; y las posiciones intermedias, dispuestas a la negociación. Si la experiencia de las mujeres en la medicina refleja esta diversidad de posturas, también es cierto que los modos concretos en que viven y reaccionan a

114 Estudos Feministas, Florianópolis, 24(1): 101-117, janeiro-abril/2016 
las paradojas y tensiones - un ejemplo clave es el de la doctora Suza - permiten pensar en el género sin esencialismos, pero como una dimensión central de esa experiencia profesional. Todo esto en pos del conocimiento, pero sobre todo de la cabal comprensión de las formas en que se desarrolla la atención de la salud y las posibilidades reales de transformarla. En lo que respecta a la gineco-obstetricia, lograr una atención que sea efectivamente libre de todo tipo de violencia.

\section{Referencias bibliográficas}

ANONIMA. "Testimonio de una médica especialista ante la violencia obstétrica institucionalizada". Anexo. Género y Salud en Cifras, v. 8, n. 3, p. 13-19. 2010.

ALMAGUER GONZÁLEZ, José Alejandro, GARCIA RAMÍREZ, Hernán, \& VARGAS VITE, Vicente. "La violencia obstétrica: una forma de patriarcado en las Instituciones de Salud". Género y Salud en Cifras, v. 8, n. 3, p. 4-20. 2010.

BENEVIDES, Regina y PASSOS, Eduardo "Humanização na saúde: un novo modismo?". Rev. Interface, v. 9, n. 17, p. 389-406. 2005.

BONET, Octavio. "Saber y Sentir: uma etnografía da aprendizagem da Biomedicina". Physis. Revista da Saúde Colectiva, v. 9, n. 1, p.123-150. 1999.

BRONFMAN, Mario y CASTRO, Roberto. "Discurso y práctica de la planificación familiar en América Latina". Saúde em Debate, v. 25, p. 61-8. 1989.

BUSCATTO, Marie y MARRY, Catherine. "Le Plafond de verre dans tous ses éclats. La feminization des professions superieures an XXme siècle". Sociologie du travail, v. 51, n. 2, p. 170-182. 2009.

CAMPERO, Lourdes et al. "Tendencias de cesáreas en relación con factores no clínicos en un centro de educación para el parto en la Ciudad de México". Salud Pública de México, v. 49, n. 2, p.118-125. 2007.

CASTRO, Roberto y LÓPEZ, Alejandra. Editores. Poder médico y ciudadanía. El conflicto social de los profesionales de la salud con los derechos reproductivos en América Latina: Avances y desafíos en la investigación regional. Montevideo: UdelaR/CRiM- UNAM, 2010.

CASTRO, Roberto y ERVITI, Joaquina. "25 años de investigación sobre Violencia Obstétrica en México", v. 19, n. 1, p. 37-42. 2014

CEPEDA, Agustina y RUSTOYBURU, Cecilia. "Medicalizar los cuerpos, la salud y la sexualidad en América Latina. A modo de introducción.". En: CEPEDA, Agustina y RUSTOYBURU, Cecilia (comps.) De las hormonas sexuadas al Viagra. Ciencia, Medicina y Sexualidad en Argentina y Brasil. Mar del Plata: EUDEM, 2014, p.9-31. 
CHALMERS, Beverley. "WHO: Appropiate technology for birth revisited". British Journal of Obstetrics and Gynaecology, v. 99, p. 709-710. 1992

DELGADO, Ana, TÁVORA, Ana y ORTIZ GÓMEZ, Teresa. "Las médicas, sus prácticas y el dilema con la feminidad". Estudios de Sociolinguística, v. 4, n. 2, p. 589-61 1. 2003.

ENCUESTA NACIONAL DE SALUD Y NUTRICIÓN 2012. "Elevada recurrencia a las cesáreas: revertir la tendencia y mejorar la calidad en el parto". Instituto Nacional de Salud Pública. Secretaría de Salud del Gobierno Federal. 2012. Disponible en: http://ensanut.insp.mx/doctos/analiticos/ Cesareas.pdf.

FAJARDO-DOLCl, Germán. et al. "Perfil epidemiológico general de las quejas médicas atendidas ante la CONAMED". Salud Pública de México, v. 51, n. 2, p. 361-371. 2009.

FOUCAULT, Michel. Historia de la sexualidad I. La voluntad de saber. México DF: Siglo XXI Editores, 2005.

FRANCO ROSALES, Adriana. "Violencia Obstétrica: martirio común para las mexicanas". CIMAC noticias. 4/6/2013. Disponible en: http://www.cimacnoticias.com.mx/node/ 63477.

FREYERMUTH, Graciela y SESIA, Paola (coords.). La muerte materna. Acciones y estrategias hacia una maternidad segura. México: Comité Promotor Maternidad sin riesgos y CIESAS, 2009.

GIRE (Grupo de Información en Reproducción Elegida) Capítulo 4: Violencia obstétrica. Omisión e Indiferencia. En: Derechos reproductivos en México. 2013. Disponible en: http://informe.gire.org.mx.

HÉRITIER, Françoise. Femenino/Masculino II. Disolver la jerarquía. México DF: Fondo de Cultura Económica, 2007.

IRIART, Celia, WAITZKIN, Howard, BREHIL, Jaime y ESTRADA, Alfredo. "Medicina social latinoamericana: aportes y desafíos". Revista Panamericana de Salud Pública, v. 12, n. 2. 2002.

JOHANISSON, Karin. Los signos. El médico y el arte de la lectura del cuerpo. Barcelona: Melusina, 2006.

KALINSKY, Beatriz. "'Ser mujer'” en trabajos fronterizos: las marcas del género". Revista de estudios de género La Ventana, v. 24, p. 229-256. 2006.

LACQUEUR, Thomas. La construcción del sexo. Cuerpo y Género de los griegos a Freud. Madrid: Ed. Cátedra, 1994.

LÓPEZ SÁNCHEZ, Oliva. El dolor de Eva. La profesionalización del saber médico en torno del cuerpo femenino en la segunda mitad del siglo XIX. México DF: UNAM-Fez Iztacala, 2010.

MINO, Fernando. "Parir con dolor. La violencia obstétrica en los servicios de salud". La Jornada, Jueves 5 de abril de

116 Estudos Feministas, Florianópolis, 24(1): 101-117, janeiro-abril/2016 
2012. Disponible en: http://www.jornada.unam.mx/2012/ 04/05/ls-central.html.

NARI, Marcela. Políticas de maternidad y maternalismo político: Buenos Aires, 1890-1940. Buenos Aires: Biblos, 2004.

POZZIO, María. "El hecho de que sean más mujeres no garantiza nada: feminización y experiencias de las mujeres en la gineco-obstetricia en México". Salud Colectiva, v.10, n. 3, p. 325-337. 2014.

PRADO, Giulio y GONZALEZ, Verónica. "La medicina social y los desafíos impostergables en Nuestra América. ALAMES 30 años". Voces en el Fénix, n.41. Disponible en: http:// www.vocesenelfenix.com/category/ediciones/n\%C2\%BA41. 2015.

RAYAS VELASCO, Lucía. Armadas: Un análisis de género desde el cuerpo de las mujeres combatientes. México DF: El colegio de México. 2009.

RELACAHUPAN- Uruguay. "Parto Humanizado: Recopilación de folletos y artículos sobre el tema. Materiales de apoyo para talleres de capacitación. 2006-2007."

ROHDEN, Fabiola. "Ginecologia, gênero e sexualidade na ciência do século XIX". Horizontes Antropológicos, v.8, n. 17, p. 101-125. 2002.

SCOTT, Joan. "Experiencia". Revista de estudios de género La Ventana, v. 13, p. 43-73. 2001.

SOMMESTAD, Lena. "Gendering Work, Interpreting Gender: the masculinization or dairy work in Sweden, 1850-1950". History Workshop, v.37, n. 1, p. 57-75. 1994.

WAGNER, Marsden. "La partería global -tradicional y oficialy la humanización del nacimiento". Midwifery Today, n. 83, autumm 2007. Traducción de Ana María Piza.

[Recebido em 14 de setembro de 2014 via portal e reapresentado em 21 de julho de 2015]

\begin{abstract}
The Gynecology Obstetrics in México: Between "Humanized Childbirth" and Obstetric Violence

Abstract: While the ob-gyn becomes feminized, the problematization of obstetric violence increases. From these two phenomena, the article aims to describe the different positions the actors have taken on the introduction of humanized birth. These positions are 'open', 'intermediate' and 'resistance' and aim to reduce the obstetrics violence. In particular, we emphasize the explanations and reactions of the ob-gyn women when they have to face violence obstetric. The analysis seeks to show how gender and anthropology construction as a method and approach contribute to understand the phenomenon and to make intelligible this apparent paradox. In conclusion, we propose that to establish relations between the feminization of the ob-gyn and the problematization of the obstetric violence allow us an entry to understand the scope and limitations of the process of feminization of the medical profession

Keywords: Gender; Medicine; Childbirth; Obstetric violence; Anthropology.
\end{abstract}

